\section{Rapid Sequence Intubation in the Pre-Hospital Setting - Difference Between Trauma and Nontrauma Patients}

\author{
KATJA LAH • MILJENKO KRIŽMARIĆ • ŠTEFEK GRMEC
}

\author{
KATJA LAH $(\triangle) \bullet$ \\ ŠTEFEK GRMEC \\ Center for Emergency Medicine \\ Ljubljanska 5. \\ 2000 Maribor, Slovenia \\ Phone: + 38623211234 \\ Fax: +3862 3331801 \\ E-mail: katka_lah@yahoo.com \\ Medical Faculty University of Maribor \\ Slomškov trg 15., 2000 Maribor, Slovenia \\ Medical Faculty University of Ljubljana \\ Vrazov trg 2., 1104 Ljubljana, Slovenia

MILJENKO KRIŽMARIĆ $\bullet$
ŠTEFEK GRMEC
Faculty for Health Sciences
University of Maribor

\begin{abstract}
Aim. To determine, in a prospective observational study, whether there are differences in the practice of rapid sequence intubation (RSI) and to ascertain the characteristics between trauma and non-trauma patients that were intubated in a prehospital setting.

Methods. Included were patients (18 years and over) who were not in cardiac arrest and who underwent RSI and were transported to hospital. From J anuary 2000 to December 2006 we intubated 636 patients in cardiac arrest, 159 critically ill non-trauma patients and 142 trauma patients. Placement of an endotracheal tube was confirmed by capnography. We compared medical and trauma groups of intubated patients. We used the two-independent sample t-test, Chi-square test and Wilcoxon-Mann Whitney test for statistical analysis.

Results. Statistical differences between groups (medical vs. trauma): initial main arterial pressure $(104.9+/-34.6$ vs. 90.7 + /- 24.8; $p=0.01)$, blood glucose levels $(9.2+/-3.5$ vs. $5.9+/-1.9 ; p=0.011)$, administration of colloids $(13,1 \%$ vs. 70,2 ; $p=0.003$ ) and Hyperhaes ( $2.5 \%$ vs.17.6 \%; $p=0.001)$, male gender ( 62.3 vs $81.6 ; p=0.014)$, rate of RSI $(71.1 \%$ vs. 96.4 $\%$; $p<0.001$ ), initial GCS distribution 3-4/5-8/9-15 (30.9\%/61.6 \% /7.5 \% vs $11.7 \% / 60,2 \% / 28,1 \% ; p<0.001$ ), initial pet $\mathrm{CO}_{2}(49,5+/-8,4 \mathrm{mmHg}$ vs. $32,8+/-5.4 \mathrm{mmHg} ; \mathrm{p}=0.007)$, APACHE II first day of hospitalization $(25,9+/-4.9 \mathrm{vs.} 20,8$ $+/-3.6 ; p=0.002)$ and hospital mortality $(78 / 159(49.1 \%)$ vs. $44 / 142(30.1 \%) ; p=0.023)$. We also analyzed the number of intubation attempts, intubation success rate, perceived difficulty of intubation and side effects with complications. The hospital survival analysis showed that survivors are younger $(54.2+/-19.9$ vs. $62.3+/-18.8 ; p=0.019)$, have a higher rate of RSI (175/179(97.7 \%) vs. 75/122(61.6\%); $p=0.002)$ and have a better (lower) APACHE II score $(19.9+/-3.6$ vs.28.3 +/4.6; $p=0.002$ ). We found the highest mortality rate in the subgroup of patients with non-traumatic intracranial hemorrhage $(58.8 \%, 60 / 102)$.

Conclusion. In non-trauma, critic ally ill patients we found a lower rate of RSI, more patients with an initial GCS of 3-4, higher APACHE II first day, higher initial pet $\mathrm{CO}_{2}$ and higher hospital mortality than in trauma patients.
\end{abstract}

Keywords: rapid sequence intubation, pre-hospital setting, injured patients, critically ill patients, prognosis

\section{Introduction}

Airway control is the first priority in managing injured (1-6) and critically ill patients. (7-16) Failure or loss of airway, with resultant failure of ventilation and oxygenation is a terminal pathway for many emergency patients. Rapid sequence intubation (RSI) is the cornerstone of emergency airway management. Furthemore, advanced techniques such as rapid sequence intubation, require a higher level of training and expertise for pre-hospital providers. (1,6,17-19) RSI performed by pre-hospital physicians is useful, effective, safe, successful and part of current standard care. (20-25) In the Centre for Emergency Medicine in 
Maribor (physician-based Emergency Medical Service (EMS)) RSI is part of a protocol for the maintance of adequate airway management in critically ill and injured patients. $(2,26)$ The majority of trials on RSI, in the pre-hospital setting, have focused on trauma patients only. The aim of our prospective observational study was to determine if there are differences between trauma and non-trauma intubated patients in the pre-hospital setting.

\section{Methods and patients}

This prospective, observational study was performed in the Centre for Emergency Medicine in Maribor, the second largest city in Slovenia with a population of approximately 200.000 spread over an area of approximately $780 \mathrm{~km}^{2}$. Since August 1999 we have had two pre-hospital emergency units (PHU). A $\mathrm{PHU}$ is an advanced life support (ALS) unit comprised of an emergency physician and two registered nurses or a nurse. A PHU is primarily routinely dispatched to the scene in emergency situations (e.g. in the case of presumed cardiac arrest, heart attack, respiratory distress, cerebrovascular incident, trauma, parturition, severe poisoning, etc.).

Adult patients (18 years and over) who were intubated by emergency physicians using the RSI method or rapid sequence induction were included in the study. RSI was performed with induction agents (midazolam, etomidate, ketamine, thiopental, fentanyl) in recommended doses, followed by the administration of a rapid-acting neuromuscular blocking agent (succinylcholine $1-1.5 \mathrm{mg} / \mathrm{kg}$ ) and/or non-depolarizating neuromuscular blocking agent (vecuronium $0.08-0.15 \mathrm{mg} / \mathrm{kg}$ ).

Endotracheal tube position was confirmed with auscultation and capnometry/capnography (the examiners looked for the characteristic $\mathrm{CO}_{2}$ waveform, value of end tidal carbon dioxide (et $\mathrm{CO}_{2}$ ) and clinical signs). After successful intubation, all patients were mechanically ventilated using the ventilator Medumat Standard WM 2250, Weinmann medical technology,
Hamburg, Germany. Between January 2000 and December 2006 we intubated 636 patients in cardiac arrest, 159 critically ill (non-trauma) patients and 142 trauma patients. 43 patients (Glasgow Coma Scale (GCS) = 3) who were intubated without any drugs for induction were excluded from the study. We compared the medical group and the trauma group of intubated patients in the pre-hospital setting. We obtained APACHE II and Mainz Evaluation Emergency Scores and MEES (difference between initial and final MEES).

Statistical analysis was performed using the two-independent sample t-test, Chi-square test, Fisher exact test and Wilcoxon-Mann Whitney test. Analysis of independent predictors for survival from univariate analysis was performed using multivariate logistic regression. P-value of less than 0.05 was considered significant. For statistical analysis we used computer software SPSS12.01 Inc.Chicago, Illionis, USA.

\section{Results}

We found significant differences between groups (medical vs. trauma) in initial main marterial pressure, glucose levels, application of colloids and Hyperhaes $(7,2 \% \mathrm{NaCl}$ and $6 \%$ HAES solution), male gender, rate of RSI, initial GCS distribution, initial exhaled carbon dioxide (pet $\mathrm{CO}_{2}$ ), APACHE II after first day of hospitalization and hospital mortality (table 1). Reasons for intubation in specific medical groups were cerebrovascular incident (102/159; 64.2\%), respiratory failure/distress (27/159;16.9 $\%)$, intoxication (12/159; $7.6 \%)$, shock (14/159; $8.8 \%)$ and other (4/159; 2.5 $\%)$. In the respiratory failure/distress and intoxication subgroups we found significantly higher initial pet $\mathrm{CO}_{2}$ and desaturation levels (average initial pet $\mathrm{CO}_{2}$ for these groups was $69.4+/-15.9$ $\mathrm{mmHg}$ and average oxygen saturation was $74.3+/-16.3 \%)$. In the trauma group we performed RSI in 93 isolated severe head injury patients, 10 maxillofacial trauma patients and 39 polytrauma patients. In 290 of 301 (96,3\%) patients intubation was successful at the first attempt. In 11 (3,7\%) patients a second attempt was necessary. Final success rate of tracheal intubation was $100 \%$. We noticed a significant difference in the number of second attempt intubations between the groups (medical group vs. trauma group; 2/159 (1,6 $\%)$ vs. $9 / 142(6,3 \%) ; p=0.03)$. Major immediate adverse events related to the procedure of RSI were: hypoxia (oxygen desaturation < $90 \%$ ) in three (1\%) patients (two patients in the medical group and one in the trauma group), hypotension (systolic blood pressure $<90 \mathrm{mmHg}$ ) in eight (2,6\%) patients (seven in medical and one in trauma patients), bradycardia in seven (2,3\%) patients (six in the medical group and one in the trauma group) and fasciculations in three $(1 \%)$ patients (two in the medical group and one in the trauma group). All in all, we found 17 (10,7\%) cases of complications in the medical group and four $(2,8 \%)$ in the trauma group $(p=0.013)$. Analysis of survival (discharge from hospital) rates in hospital show that survivors are younger 54.2 +/- 19.9 vs. $62.3+/-18.8 ; p=0.019$, have a high rate of RSI (175/179 (97.7 \%) vs. 75/122 (61.5\%; $p=0.002)$, have a better (lower) APACHE II score (19.9 + - 3.6 vs. $28.3+/-4.6 ; p=0.002)$, have better (higher) MEES (22.4 +/- 3.6 vs. $16.4+/-2.4 ; p=0.02)$, have lower blood glucose levels (5.6+/- 2.5 vs. $12.3+/$ $4.3 \mathrm{mmol} / \mathrm{l} ; \mathrm{p}=0.001)$ and higher final pet $\mathrm{CO}_{2}(39.8+/-4.1$ vs. $32.1+/-3.8$; $p=0.026)$. We found the highest mortality rate in a subgroup of non-traumatic patients with intracranial hemorrhage (58.8; 60/102). Using multivariate analysis we found that independent predictors for survival were: non-traumatic intracranial hemorrhage $(\mathrm{OR}=0.21$; $95 \% \mathrm{C}$ index (Cl) $0.11-0.42 ; \mathrm{p}=0.02)$, APACHE II = or > 20 (OR 0.13; $95 \%$ $\mathrm{Cl} 0.08-0.29 ; p=0.01)$, final pet $\mathrm{CO}_{2}$ $>30 \mathrm{mmHg}$ (OR 1.31; $95 \% \mathrm{Cl} 1.13$ $-1.82 ; \mathrm{p}=0.02)$ and rate of $\mathrm{RSI}$ (OR $1.42 ; 95 \% \mathrm{Cl} 1.25-1.76 ; p=0.01$ ).

\section{Discussion}

The use of RSI was introduced to the pre-hospital environment in hope of improving patient outcome by enhancing early definitive airway management. 
Table1. Clinical and demographical characteristics of patients which underwent RSI - difference between medical and trauma group.

\begin{tabular}{|c|c|c|c|}
\hline & Medical group & Trauma group & $p$-value \\
\hline Age (years) & & & \\
\hline Gender / male & $99 / 159$ (62,3 \%) & $116 / 142(81,6 \%)$ & 0.014 \\
\hline MAP $(\mathrm{mmHg})$ & $104,9+/-34.6$ & $90.7+/-24.8$ & 0.01 \\
\hline Blood sugar (mmol/L) & $9.2+/-3.5$ & $5.9+/-1.9$ & 0.011 \\
\hline $\begin{array}{l}\text { Arrival time on the scene } \\
\text { (min) }\end{array}$ & $6.9+/-3.6$ & $7.4+/-3.2$ & 0.75 \\
\hline On-scene time (min) & $26,9+/-9,3$ & $30,6+/-8,6$ & 0.36 \\
\hline Applications of colloids & $21 / 159(13,2 \%)$ & $100 / 142(70,4 \%)$ & 0.003 \\
\hline Application of Hyperhaes & 4/159 (2,5\%) & $25 / 142(17,6 \%)$ & 0.001 \\
\hline $\begin{array}{l}\text { Second attempt of intuba- } \\
\text { tion }\end{array}$ & 2/159 (1.6 \%) & 9/142 (6.3 \%) & 0.03 \\
\hline $\begin{array}{l}\text { Immediate adverse events } \\
\text { of RSI }\end{array}$ & $17 / 159(10,7 \%)$ & $4 / 142(2,8 \%)$ & 0.013 \\
\hline $\begin{array}{l}\text { Initial GCS (3-4 / 5-8 / 9-15) } \\
\text { distributions }\end{array}$ & $\begin{array}{l}30.9 \% / 61.6 \% / \\
7.5 \%\end{array}$ & $\begin{array}{l}11,7 \% / 60.2 \% / \\
28.1 \%\end{array}$ & $<0.001$ \\
\hline Initial $\mathrm{SaO}_{2}(\%)$ & $78.9+/-11.7$ & $86.9+/-9.4$ & 0.04 \\
\hline Initial MEES & $18.6+/-2.8$ & $22.8+/-2.4$ & 0.02 \\
\hline$\triangle$ MEES & $1.91+/-0.31$ & 3.69. +/- 0.82 & 0.03 \\
\hline APACHE ॥ & $25,9+/-4.9$ & $20.8+/-3.6$ & 0.002 \\
\hline Hospital mortality & 78/159 (49,1\%) & $44 / 142(30,1 \%)$ & 0.04 \\
\hline Rate of RSI & $110 / 159(71.1 \%)$ & 140/142 (96.4 \%) & 0.04 \\
\hline Initial pet $\mathrm{CO}_{2}(\mathrm{mmHg})$ & $49.5+/-8.4$ & $32.8+/-5.4$ & 0.008 \\
\hline Final pet $\mathrm{CO}_{2}(\mathrm{mmHg})$ & $32.6+/-5.6$ & $40.4+/-4.8$ & 0.035 \\
\hline
\end{tabular}

GCS = Glasgow Coma Scale, MAP = mean arterial pressure, RSI = rapid sequence intubation, pet $\mathrm{CO}_{2}=$ exhaled carbon dioxide, $\mathrm{SaO} 2=$ arterial oxygen saturation

RSI performed by emergency physicians improves patient's outcome and is a safe procedure. $(2,19-22,25)$ How- of limited knowledge of drug kinetics and pharmacodynamics. (1,27-29) Therefore, the value of rapid sequence induction for intubation does depend on each EMS design and their ability to establish personnel requirements and ongoing training expertise in airway management skills, medical direction and supervision. $(1,16,23)$ In the available literature we found limited studies on RSI in non-trauma patients in the pre-hospital setting. $(7,8,10,15)$ One reason for this is that the paramedicbased system rarely uses RSI in the pre-hospital setting in non-trauma patients. (30) The results of our trial show that non-trauma patients are initially in a worse condition (patients are older, have worse initial GCS, MEES and APACHE scoring, higher initial pet $\mathrm{CO} 2$ and lower oxygen saturation). Higher initial pet $\mathrm{CO} 2$ and desaturation suggest a primary asphyxial mechanism underlying the clinical problem. The starting point is the pathophysiology of chronic obstructive pulmonary disease (COPD), asthma or heart failure with pulmonary edema, where as secondary problem is the airway in unconscious patients (especially in the respiratory and intoxication subgroup of non-trauma patients). (31) In non-trauma patients we found high proportions of GCS 3-4 with a logical lower rate of RSI (intubations without drugs or muscle relaxation). This group of patients has a significantly lower $\triangle$ MEES. In general, $\triangle$ MEES $=$ or $>$ 2 signifies an improvement in clinical condition. In our trial we demonstrated a significant difference in improvement between groups, with an initially worse condition and limited effect of treatment in the pre-hospital setting (short time of care and limited diagnostic resources). (32) On the other hand we found significantly lower initial mean arterial pressure (MAP) and significantly higher rate of application of colloids and Hyperhaes in trauma group patients. In the trauma group of our trial, hypotension was an important initial problem and RSI medication had to be adapted. $(1,2)$ Medication also had to be used in cases of heart failure and COPD/asth- 
ma patients. $(7,8,10,30)$ These results confirm the necessity of diagnosis-based protocols for RSI. (10-12)

All 301 patients (100\%) underwent successful RSI, 290 (97\%) in the first attempt. This result is better than the results we found in the literature. (11-14,16-19,24,25,33-35) However, the group comparison showed a significantly higher number of second attempts in trauma patients, especially with maxillofacial and head trauma. The problem was visualization of the vocal cords because of hemorrhage and changes of airway anatomy (obstruction), which both represent advanced challenges for intubation in the field. $(1,3,6)$

In the analysis of major immediate adverse events in the procedure of RSI we found a significantly higher level of complications in the non-trauma group. The most frequently recorded adverse events were hypotension and bradycardia. Complications are related to the underlying pathology and the age of non-trauma patients. Overall we confirmed immediate adverse events in $6,9 \%$ of patients. The rate of immediate complications in the literature is between $3 \%$ - $35 \%$. $(13,36,37)$ In our trial we had only 3 cases of procedural hypoxia (two in the medical and one in the trauma group). All 3 cases were in the subgroup of 'second attempt'. This observation is similar to the observation of Levitan, et. all. (38) Procedural hypoxia is in correlation with repeated laryngoscopy and the time needed to intubate. Our good results are most likely a consequence of good pre-oxygenation and quick and effective laryngoscopy and insertion of the endotracheal tube.

Hospital mortality was significantly higher in the medical group. In a multivariate analysis of independent factors for survival we discovered that APACHE $\mathrm{II}$, final pet $\mathrm{CO}_{2}$ and rate of $\mathrm{RSI}$ are independent variables for survival. Rates of $\mathrm{RSI}$ and survival were higher in trauma patients, and maybe this correlation suggests a benefit of RSI regardless of GCS score. $(27,32)$ In the medical group physicians frequently intubated without medications and did not use succinylcholine-assisted intubation. This was due to a large number of patients having an initial GCS 3-4. It is difficult to evaluate what the primary reason for the better survival rate in trauma patients is due to: initially higher GCS or RSI as a method of intubation (the values of APACHE II suggest an initially better condition).

One of the most interesting findings of our trial is the importance of pet $\mathrm{CO}_{2}$ for survival. The values of final pet $\mathrm{CO}_{2}>$ $30 \mathrm{mmHg}$ were in correlation with survival. Davis et al found low pet $\mathrm{CO}_{2}$ in 35
$\%$ of patients in their trial in San Diego and attributed this finding to inadvertent hyperventilation. (39) In our trial all patients were mechanically ventilated after $\mathrm{RSI}$ according to recommendations, and in these circumstances the probability for inadvertent hyperventilation is minimized. We practiced moderate hyperventilation only in severe head injury patients with evident signs of higher intracranial pressure. Pet $\mathrm{CO}_{2}$ is probably in correlation with MAP and cardiac output (in the medical group we confirmed a significantly lower MAP and final pet $\left.\mathrm{CO}_{2}\right)$. $(40,41)$ The predicting value of pet $\mathrm{CO}_{2}$ is confirmed in studies about patients in cardiac arrest (42) and trauma patients. (43)

\section{Conclusion}

In comparing medical and trauma patients intubated in the field we discovered that these two groups really differ in many parameters. The patients in the medical group were in a worse initial condition, had a worse prognosis and a higher rate of major immediate adverse events during the procedure of RSI (most frequent being hypotension and bradycardia).

Among the trauma patients we found more second attempts of RSI and problems with visualization.

These results confirm the necessity of diagnosis-based protocols for RSI. 


\section{REFERENCES}

1. Ummenhofer W, Scheidegger D. Role of the physician in prehospital management of trauma: European perspective. Curr Opin Crit Care 2002;8:559-65.

2. Klemen $\mathrm{P}, \mathrm{Grmec}$ Š. Effect of prehospital advanced life support with rapid sequence intubation on outcome of severe traumatic brain injury. Acta Anaesthesiol Scand 2006;50:1250-4.

3. Lewis RJ. Prehospital care of the multiply injured patient-The challenge of figuring out what works. JAMA 2004;291(11):1382-4.

4. Davis DP, Kene M, Vilke GM, Sise MJ, Kennedy F, Eastman AB, et al. Head - injured patients who" talk and die": the San Diego perspective. J Trauma 2007;62:277-81.

5. Di Bartolomeo S, Sanson G, Nardi G, Michelutto V, Scian E. Inadequate ventilation of patients with severe brain injury: a possible drawback to prehospital advanced trauma care? Eur J Emerg Med 2003;10:268-71.

6. Fakhry SM, Scanlon JM, Robinson L, Askari R, Watenpaugh RL, Fata P, et al. Prehospital rapid sequence intubation for head trauma: conditions for a successful program. J Trauma 2006;60(5):997-1001.

7. Reynolds SF, Heffner J. Airway management of the critically ill patient: rapid-sequence intubation. Chest 2005;127:1397-412.

8. Kraus JJ, Metzler MD, Coplin WM. Critical care issues in stroke and subarachnoid hemorrhage. Neurol Res 2002;24(suppl 1):S47-57.

9. Ricard-Hibon A, Chollet C, Leroy C, Marty J. Succinylcholine improves the time of performance of a tracheal intubation in prehospital critical care medicine. Eur J Anaesthesiol 2002;19:361-7.

10. Adnet F, Minadeo JP, Finot MA, Borron SW, Fauconnier V, Lapandry C, et al. A survey of sedation protocols used for emergency endotracheal intubation in poisoned patients in the French prehospital medical system. Eur J Emerg Med 1998;5(4):415-9.

11. Marvez-Valls E, Houry D, Ernst AA, Weiss SJ, Killen J. Protocol for rapid sequence intubation in pediatric patients - a four-year study. Med Sci Monit 2002;8:CR229-34.

12. Marvez E, Weiss SJ, Houry DE, Ernst AA. Predicting adverse outcomes in a diagnosis based protocol system for rapid sequence intubation. Am J Emerg Med 2003;21(1):23-9.

13. Simpson J, Munro PT, Graham CA. Rapid sequence intubation in the emergency department: 5 year trends. Emerg Med $J$ 2006;23(1):54-6.

14. Stevenson AG, Graham CA, Hall R, Korsah P, McGuffie AC. Tracheal intubation in the emergency department: the Scottish district hospital perspective. Emerg Med J 2007; 24(6):394-7.

15. Alanoglu Z, Ates Y, Yilmaz AA, Tüzüner F. Is there an ideal approach for rapid-sequence induction in hypertensive patients? J Clin Anesth 2006;18(1):34-40.

16. Jeremie N, Seltzer S, Lenfant F, Ricard-Hibon A, Facon A, Cabrita B, et al. Rapid sequence induction: a survey of practices in three French prehospital and mobile emergency units. Eur J Emerg Med 2006;13(3):148-55.

17. Bochicchio GV, Scalea TM. Is field intubation useful? Curr Opin Crit Care 2003;9(6):524-9.

18. Di Bartolomeo S, Sanson G, Nardi G, Scian F, Michelutto V, Lattuada L. Effects of 2 patterns of prehospital care on the outcome of patients with severe head injury. Arch Surg 2001;136(11):1293-300.

19. Combes X, Jabre P, Jbeili C, Leroux B, Bastuji-Garin S, Margenet A, et al. Prehospital standardization of medical airway management: incidence and risk factors of difficult airway. Acad Emerg Med 2006;13(8):823-34.

20. Kill C, Wranze E, Wulf H, Geldner G. Rapid sequence induction in prehospital emergency medicine: it is safe? Anaesthesiol Intensivmed Notfallmed Schmertzher2004;39(11):668-71.

21. Ricard Hibon A, Chollet C, Belpomme V, Duchateau FX, Marty J. Epidemiology of adverse effects of prehospital sedation analgesia. Am J Emerg Med 2003;21(6):461-6.

22. Mackay CA, Terris J, Coats TJ. Prehospital rapid sequence induction by emergency physicians: is it safe? Emerg Med J 2002;19(4):374.

23. Davis BD, Fowler R, Kupas DF, Roppolo LP. Role of rapid sequence induction for intubation in the prehospital setting: helpful or harmful? Curr Opin Crit Care 2002;8(6):571-7.

24. Sloane C, Vilke GM, Chan TC, Hayden SR, Hoyt DB, Rosen P. Rapid sequence intubation in the field versus hospital in trauma patients. J Emerg Med 2000;19(3):259-64.

25. Sing RF, Rotondo MF, Zonies DH, Schwab CW, Kauder DR, Ross SE, et al. Rapid sequence induction for intubation by an aeromedical transport team: a critical analysis. Am J Emerg Med 1998;16(6):598-602.

26. Grmec Š, Mally Š. Prehospital determination of tracheal tube placement in severe head injury. Emerg Med J 2004;21:518 -52.

27. Davis DP, Vadeboncoeur TF, Ochs M, Poste JC, Vilke GM, Hoyt DB. The association between field Glasgow Coma Scale score and outcome in patients undergoing paramedic rapid sequence intubation. J Emerg Med 2005;2984:391-7.

28. Bernard SA. Paramedic intubation of patients with severe head injury: a review of current Australian parctice and recommendation for change. Emerg Med Australas 2006; 18(3):221-8.

29. Davis DP, Stern J, Sise MJ, Hoyt DB. A follow-up analysis of factors associated with head-injury mortality after paramedic rapid sequence intubation. J Trauma 2005;59(2): 486-90. 
30. Stiell IG, Spaite DW, Field B, Nesbitt LP, Munkley D, Maloney J, et all. Advanced life support for out-of-hospital respiratory distress. N Engl J Med 2007;356:2156-64.

31. Grmec Š, Lah K, Tusek -Bunc K. Difference in end-tidal CO2 between asphyxia cardiac arrest and ventricular fibrillation/pulseless ventricular tachycardia cardiac arrest in the prehospital setting. Crit Care 2003;7:R139-44.

32. Grmec Š, Gašparović V. Comparison of APACHE II, MEES and Glasgow Coma Scale in patients with non-traumatic coma for prediction of mortality. Crit Care 2001;5:19-23.

33. Wang HE, Yealy DM. How many attempts are required to accomplish out-of-hospital endotracheal intubation. Acad Emerg Med 2006;13(4):372-7.

34. Jennet Me, Kendal KM, Fourre MW, Burton JH. Unrecognized misplacement of endotracheal tubes in a mixed urban to rural emergency medical services setting. Acad emerg Med 2003;10(9):961-5.

35. Timmermann A, Russo SG, Eich C, Roessler M, Braun U, Rosenblatt WH, Quintel M. The out-of-hospital esophageal and endobronchial intubations performed be emergency physicians. Anesth Analg 2007;104:619-23.

36. Dibble C Maloba M. Rapid sequence induction in the emergency department by emergency personnel. Emerg Med J 2006;23:62-4.

37. Reid C, Chan L, Tweeddale M. The who, where and what of rapid sequence intubation prospective observational study of emergency RSI outside the operating theatre. Emerg Med J 2004,2:296-301.

38. Levitan RM, Dominici P, O'Malley G. Hypoxia during emergency department intubation: relationship to repeat laryngoscopy and time to intubation. Acad Emerg Med 2006;13(Suppl 1):166.

39. Davis DP, Ochs M, Hoyt DB, Marshall LK, Rosen P. The San Diego paramedic rapid sequence intubation trial: a three-year experience. Acad Emerg Med 2003;10(5):446.

40. Isserles Sa, Bree Ph. Can changes in end-tidal pCO2 measure changes in cardiac output Anest Analg 1991;73:808-14.

41. Shibutani K, Muraoki M, Shirasaki S, Kubal K, Sanchala VT, Gupte P. Do changes in end tidal pCO2 quantitatively reflect changes in cardiac output. Anesth Analg 1994;79:829-33.

42. Grmec Š, Križmarić M, Mally S, Koželj A, Špindler M, Lešnik B. Utstein-style analysis of out-of-hospital cardiac arrest -bystander CPR and end-expired carbon dioxide. Resuscitation 2007;72:404-14.

43. Deakin CD, Sado DM, Coats TJ, Davies G. Prehospital end-tidal carbon dioxide concentration and outcome in major trauma. J Trauma 2004;57:65-8. 